
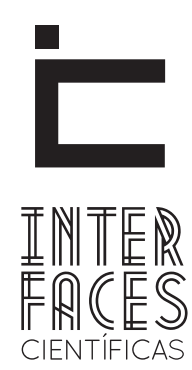

SAÚDE E AMBIENTE

\title{
AVALIAÇÃO DOS NIVEIS PLASMÁTICOS DE IGF-I, GLICOSE E INSULINA NO PRÉE PÓS-OPERATÓRIO PRECOCE DE PACIENTES OBESOS SUBMETIDOS À CIRURGIA DE GASTROPLASTIA E DERIVAÇÃO EM Y DE ROUX POR LAPAROSCOPIA
}

Paulo Autran Leite Lima ${ }^{1}$

Juliana Silva Moura ${ }^{3}$
Fábio Almeida²

Antônio Alves Júnior ${ }^{4}$

\section{RESUMO}

Este estudo teve como objetivo avaliar o comportamento dos níveis plasmáticos de IGF-I, glicose e insulina no pré e pós-operatório precoce de pacientes obesos submetidos à cirurgia de gastroplastia e derivação em Y de Roux por laparoscopia. Foi coletado amostra sanguínea de 10 indivíduos no pré-operatório e após 45 dias do procedimento cirúrgico, sendo analisadas alterações de IGF-I, glicose, insulina basal, IMC e peso. Foi observado que após 45 dias houve redução de $23,9 \%$ dos níveis de IGF-I,
$10,3 \%$ dos valores glicêmicos, $83,9 \%$ dos níveis de insulina, de $25,5 \%$ do IMC e de $25,6 \%$ do peso. Esses dados sugerem que, após 45 dias da cirurgia de derivação gástrica em $Y$ de Roux por laparoscopia, os parâmetros dos níveis de IGF-I, glicose e insulina basal tendem a baixar.

\section{PALAVRAS-CHAVE:}

Obesidade Mórbida. IMC. IGF-I. Glicemia. Insulina. 


\section{ABSTRACT}

This study aimed to evaluate plasma levels of IGF-I, glucose and insulin in the pre-and early postoperative of obese patients undergoing bariatric surgery and Roux-Y bypass through laparoscopy. It was collect blood samples of 10 patients preoperatively and 45 days after the surgery, verifying any changes in IGF-I, glucose, insulin, as well as the follow up of BMI and weight. It was observed a reduction of $23.9 \%$ in IGF-I, $10.3 \%$ in the glucose, $83.9 \%$ in insulin levels in addition to a decrease of $25.5 \%$ in the BMI and $25.6 \%$ in the weight after 45 days of surgery. These data suggest that after 45 days of gastric bypass surgery and Roux-Y bypass through laparocopy, the levels of IGF-I, glucose and insulin tend to decrease.

\section{KEYWORDS}

Morbid obesity. BMI. IGF-I. Glucose. Insulin.

\section{RESUMEN}

Esto estudio tuvo como objetivo evaluar el comportamiento de los niveles plasmáticos de IGF-I, glucosa e insulina en el pre y postoperatorio precoz de pacientes obsesos sometidos a la cirugía de gastropatía y derivación en Y de Roux por laparoscopia. Fue recogida muestra sanguínea de 10 individuos en el preoperatorio y después de 45 días del procedimiento quirúrgico, siendo analizadas alteraciones de IGF-I, glucosa, insulina, IMC y peso. Fue observado que después de 45 días de cirugía bariátrica hubo reducción de $23,9 \%$ de IGF-I, $10,3 \%$ de la glucosa, $83,9 \%$ de la insulina, 25,5\% del IMC e 25,6\% del peso. Esos datos sugieren que, después de 45 días de la cirugía de derivación gástrica en $Y$ de Roux por laparoscopia, los parámetros de los niveles de IGF-I, glucosa e insulina tienden bajar.

\section{PALABRAS CLAVE}

Obesidad mórbida. IMC. IGF-I. Glucemia. Insulina.

\section{INTRODUÇÃO}

Mundialmente e nas diversas faixas etárias de ambos os sexos, a prevalência da obesidade vem crescendo, o que, também, se acompanha com o aumento da mortalidade, sendo descrito como um problema de saúde pública (NOWICKI, et al, 2009).

A sua frequência varia conforme o sexo, a faixa etária, a raça e a condições sócio-econômicas. Dados epidemiológicos apontam um crescimento na prevalência na maioria dos países, seja desenvolvido ou em desenvolvimento (PORTO, et al, 2002).
A obesidade mórbida ou severa é definida por um IMC maior que $35 \mathrm{~kg} / \mathrm{m}^{2}$ associado a co-morbidades ou por um IMC maior ou igual a $40 \mathrm{~kg} / \mathrm{m}^{2}$. Em tais condições, existe maior risco de morbidade e mortalidade por problemas cardiovasculares, respiratórios, digestivos, hepáticos, metabólicos e endócrinos, reprodutivos, ortopédicos, dermatológicos, neurológicos e psicossociais (BROLIN, 2002; GOHIER, RICHARD-DEVANTOY, DENĖS 2010).

O hormônio do crescimento (GH) exerce um papel importante, não só na regulação do crescimento 
somático, mas, também, na regulação de vários processos metabólicos. Adultos com deficiência de GH apresentam aumento de massa de gordura corporal e redução da massa magra, em relação a adultos normais, com importantes consequências metabólicas no perfil lipídico e glicídico (CONCEIÇÃO, et al, 2003); (MAURAS, et al, 2000).

O eixo GH/IGF-1 está relacionado à resistência insulínica, aos fatores de risco cardiovasculares e com a fisiopatologia da síndrome metabólica. Além disso, o estado de hiperinsulinemia pode causar aumento da fração livre de IGF-1 e contribuir na fisiopatologia da lesão de órgãos alvo, associada à síndrome metabólica (BARRETO-FILHO, et al, 2005; LEAL-GUADARRAMA, OCHOA-ROJAS, MÉNDEZ, 2003).

Em pacientes obesos, o tecido adiposo atua aumentando a demanda por insulina, criando resistência a esta, ocasionando aumento na glicemia e consequente hiperinsulinemia. Em alguns casos, essa resistência pode ser atribuída à diminuição na concentração de receptores de insulina ou em falha no mecanismo de trânsito celular (GELONEZE, PAREJA, 2006; HALPERN, RODRIGUES, COSTA, 2004).

Após as primeiras opções terapêuticas terem sido efetivadas, a maioria dos pacientes perde peso, porém recupera-o em um período de cinco anos, ocasionando uma flutuação do peso corpóreo, aumentando o risco de mortalidade por complicações cardiovascu- lares. Com isso, o tratamento cirúrgico tem sido utilizado como uma alternativa para a redução do aporte calórico e, possui bons resultados a longo prazo para os obesos mórbidos (MANCINI, HALPERN, 2006; MAGGARD, et al, 2005).

Dentre as várias técnicas cirúrgicas realizadas para o tratamento da obesidade, a cirurgia de gastroplastia com derivação gastrojejunal em $Y$ de Roux possui um mecanismo de acção mista (disabsortiva e restritiva) sendo considerada padrão ouro para o tratamento da obesidade severa (TORPY, BURKE, GLASS, 2005). Alguns autores demonstraram que essa cirurgia reduz cerca de $60 \%$ do peso com taxas de resolução ou melhora das co-morbidades (BUCHWALD, et al, 2004). $\mathrm{Na}$ abordagem laparoscópica, o procedimento cirúrgico tem como suas maiores vantagens a redução da morbidade operatória e o tempo de recuperação pós-operatória, em virtude de ser menos invasiva (COHEN, et al, 2003).

Até o momento, poucos trabalhos têm analisado a temática da relação entre os níveis plasmáticos de IGF1 , glicose e insulina com obesos mórbidos no pré e no pós-operatório precoce. Este fato suscita o interesse e a necessidade de desenvolver pesquisa nessa área do conhecimento. Com isso, o objectivo desse estudo foi avaliar o comportamento dos níveis plasmáticos de IGF-1, glicose e insulina no pré e no pós-operatório precoce de pacientes obesos submetidos à cirurgia de gastroplastia e derivação em $Y$ de Roux por laparoscopia.

\section{MATERIAL E MÉTODOS}

Tratou-se de um estudo de carácter descritivo e prospectivo, sendo a coleta de dados efetuada com base em pesquisa do tipo exploratória, com acompanhamento de 10 indivíduos (5 mulheres/5 homens) considerados obesos mórbidos que tiveram indicação para realização da cirurgia bariátrica, por meio da mensuração do IMC, por diagnóstico clínico e exames laboratoriais que confirmassem tal condição. A faixa etária foi entre 30 e 50 anos, com média de 39,4 anos. 
Foram adotados os seguintes critérios de exclusão: desistência ou recusa em participar da pesquisa; identificação de alguma patologia ou condição clínica associada (câncer, coma, lesão neurológica, doença grave ou terminal, diabetes) antes e após o início da pesquisa, presença de doença cárdio-respiratória ou ósteo-articular incapacitante; presença de confusão mental ou dificuldade de diálogo e entendimento. A pesquisa foi aprovada após apreciação do Comitê de Ética e Pesquisa da Universidade Federal de Sergipe (CAAE 0918.0.000.107-06), de acordo com a Resolução 196/96 do Conselho Nacional de Saúde.

\section{RESULTADOSE DISCUSSÃO}

Atualmente, a obesidade é considerada um problema de saúde pública por provocar graves consequências sociais, físicas e psicológicas. Sua fisiopatologia é complexa e de difícil identificação, uma vez que a obesidade é caracterizada como uma doença multifatorial de complexa interacção entre factores comportamentais, culturais, genéticos, fisiológicos e ambientais (MOTA, ZANESCO, LEPTINA, 2007).

Várias modificações fisiológicas e metabólicas ocorrem em pacientes obesos, dentre elas alterações hormonais significativas. Alguns autores observaram que o indivíduo obeso tente a possuir níveis baixos de IGF-1 e elevados índices de glicemia e insulina (FEITOSA, et al, 2007; GELONEZE, et al, 2001).

O presente trabalho acompanhou o comportamento dos níveis de IGF-1, glicemia e insulina no pré e no pós-operatório precoce de pacientes submetidos à cirurgia de Fobi-Capella, com intuito de acompanhar o papel desses elementos na fisiopatologia da obesidade e fornecer subsídios para o tratamento e prevenção dessa patologia.
Todos os participantes da pesquisa foram orientados quanto ao jejum de 12 horas no dia anterior a coleta de sangue. As amostras foram colhidas em tubo de ensaio seco, sendo o soro tratado com EDTA. As substâncias pesquisadas foram IGF-1, glicose e insulina por meio dos métodos IRMA (imunorradioensaio), enzimático-colorimétrico e quimioluminescencia, respectivamente.

A técnica cirúrgica empregada nos pacientes foi a gastroplastia vertical com anel associada à derivação gastrointestinal em Y de Roux (Fobi-Capella) via laparoscopia (BUCHWALD, et al, 2004; HALPERN et al, 2006; MARTINS, 2005).

Com relação ao IMC, foi observado que após 45 dias da cirurgia bariátrica $\left(38,0 \pm 4,7 \mathrm{Kg} / \mathrm{m}^{2}\right)$ houve redução de 25,5\% em comparação ao período pré-operatório $\left(51,0 \pm 9,5 \mathrm{Kg} / \mathrm{m}^{2}\right), p<0,05$. Essa redução do IMC foi inferior a encontrada por Engstrom e outros autores (2006) que relataram em seu estudo uma diminuição de $28,9 \%\left(45,0 \pm 6,0 \mathrm{Kg} / \mathrm{m}^{2}\right.$ para $\left.32,0 \pm 6 \mathrm{Kg} / \mathrm{m}^{2}\right)$ e por Geloneze outros autores (2001), onde a redução do IMC foi de $36 \%\left(54,1 \pm 9,1 \mathrm{Kg} / \mathrm{m}^{2}\right.$ para $34,6 \pm 6,3 \mathrm{Kg} /$ $\mathrm{m}^{2}$ ). Essa diferença pode ser explicada pelo fato de que esses autores observaram a redução do IMC por um tempo maior de pós-operatório.

Fato semelhante aconteceu com o peso desses indivíduos, reduzindo de $147,5 \pm 37,3 \mathrm{Kg}$ para $109,7 \pm 18,6 \mathrm{Kg}, p<0,05$. Essa redução assemelha-se ao encontrado por Freire (2006), que relatou uma porcentagem média de perda de peso de $21,0 \%$ com a técnica bypass gástrico nesse mesmo período de avaliação. Essa redução de peso decorreu da acção restritiva da técnica cirúrgica empregada, que diminui sensivelmente a quantidade de alimento necessário para proporcionar a saciedade requerendo adaptação 
do padrão de comportamento alimentar. Os valores médios da avaliação laboratorial dos indivíduos são apresentados no Quadro 1.

Quadro 1 - Características dos pacientes no pré-operatório (Pré) e no $45^{\circ}$ dia de pós-operatório (Pós-45) (valores em média \pm desvio padrão)

\begin{tabular}{llll}
\hline & \multicolumn{1}{c}{ Pré } & \multicolumn{1}{c}{ Pós-45 } & \multicolumn{1}{c}{ p } \\
\hline Peso $(\mathrm{Kg})$ & $147,5 \pm 37,3$ & $109,1 \pm 18,6$ & $<0,05$ \\
IMC $\left(\mathrm{Kg} / \mathrm{m}^{2}\right)$ & $51,0 \pm 9,5$ & $38,0 \pm 4,7$ & $<0,05$ \\
IGF-I $(\mathrm{ng} / \mathrm{mL})$ & $193,3 \pm 64,7$ & $147,1 \pm 85,2$ & $>0,05$ \\
Glicemia $(\mathrm{mg} / \mathrm{dL})$ & $96,4 \pm 26,7$ & $86,5 \pm 8,9$ & $>0,05$ \\
Insulina $(\mathrm{uU} / \mathrm{mL})$ & $55,4 \pm 31,2$ & $8,9 \pm 5,0$ & $<0,05$ \\
\hline
\end{tabular}

$\mathrm{p}=$ nível de significância.

Na Figura 1 estão referenciados as médias dos índices de IGF-1 e seus respectivos desvios padrões. Foi observado que, apesar da queda dos níveis de IGF1 após 45 dias de pós-operatório $(193,3 \pm 64,7 \mathrm{ng} / \mathrm{mL}$ para $147,1 \pm 85,2 \mathrm{ng} / \mathrm{mL}$ ), não houve diferença estatística entre os grupos, $p>0,05$. Esse estudo corrobora com Freire (2006) que avaliou o índice antes e após a cirurgia bariátrica. Essa autora observou, com semeIhante metodologia, que após 45 dias de procedimento cirúrgico houve uma redução do índice em $27,8 \%$. No entanto, relatou a necessidade de realização de novas pesquisas no pós-operatório tardio para que fosse avaliado o comportamento dos níveis de IGF-1.
Figura 1 - Representação gráfica da média e o desvio padrão do IGF-I entre os grupos pré e pós-45 ( $p>0,05)$.

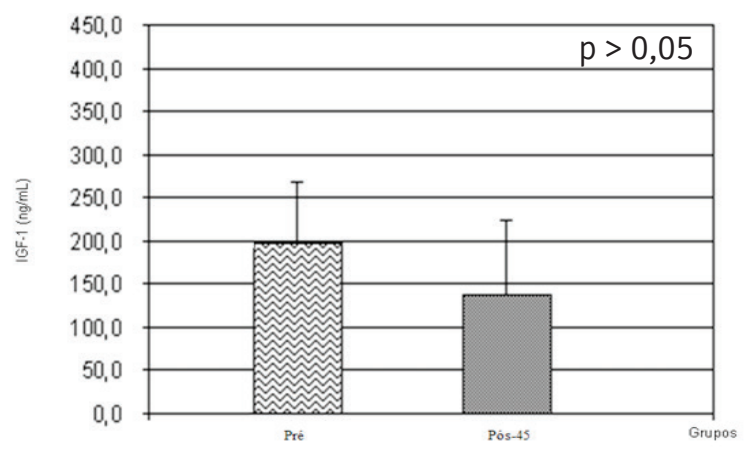

Engstrom e outros autores (2006) estudaram os efeitos do bypass gástrico nos níveis sanguíneos de GH e IGF-1 nos obesos mórbidos em comparação a deficiência do $\mathrm{GH}$ e observaram aumento dos níveis desses hormônios no pós-operatório após seis meses em mulheres e após 12 meses em ambos os sexos, associado a redução do IMC. Com isso, foi concluído que a diminuição do peso restaurou parcialmente a secreção de GH.

Os índices glicémicos apresentaram valores médios dentro dos níveis considerados normais em ambos os grupos. Apesar de ocorrer redução desses índices após $045^{\circ}$ dia pós-cirúrgico $(96,4 \pm 26,7 \mathrm{mg} /$ $\mathrm{dL}$ para $86,5 \pm 8,9 \mathrm{mg} / \mathrm{dL}$ ) não houve diferença estatística. Esse resultado corrobora com os encontrados por Frühbeck e outros autores (FRÜBECK, et al, 2004), que demonstraram que, logo após a cirurgia bariátrica de gastroplastia com derivação gastrojejunal, houve redução dos níveis glicémicos.

Acredita-se que essa melhora possa não estar atribuída ao emagrecimento ou à melhora da resistência da insulina. Porém, uma possível explicação seria a imediata e severa privação de nutrientes que ocorre após a cirurgia. Esse estado levaria a uma redução brusca dos níveis de glicemia, diminuindo assim a glicotoxicidade sobre as células pancreáticas (JAUNOO, 
SOUTHALL, BARIATRIC, 2010). A Figura 2 apresenta as médias e os desvios padrões da glicemia nos grupos.

Figura 2 - Representação gráfica da média e o desvio padrão da glicemia entre os grupos pré e pós-45 ( $p>0,05)$.

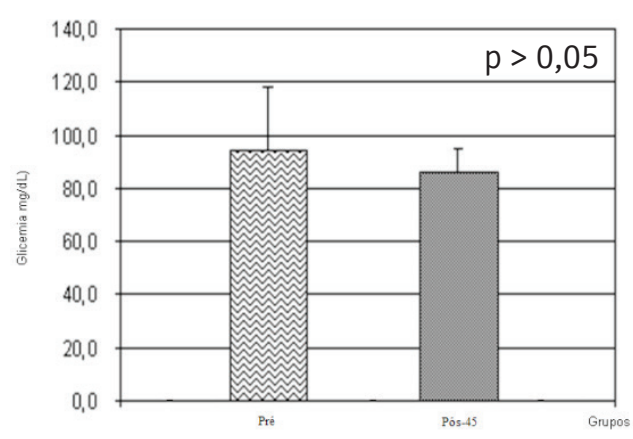

Quanto aos níveis de insulina, notou-se que após 45 dias de procedimento $(8,9 \pm 5,0 \mathrm{uU} / \mathrm{mL})$ houve uma média estatisticamente diferente quando comparado aos níveis no pré-operatório $(55,4 \pm 31,2 \mathrm{uU} /$ $\mathrm{mL}), p<0,05$. Geloneze e outros autores (2001), acompanharam, por um ano, pacientes submetidos à cirurgia bariátrica e observaram redução da resistência à insulina juntamento com melhora de parâmetros metabólicos. Segundo esses autores, a perda de peso pós-operatória induziu a uma melhora metabólica relacionada à redução na resistência insulínica devido regulação na tolerância de glicose, normalizando o diabetes. A Figura 3 apresenta as médias e os desvios padrões da variável insulina nos grupos.

Figura 3 - Representação gráfica da média e o desvio padrão da insulina entre os grupos pré e pós-45 $(p<0,05)$.

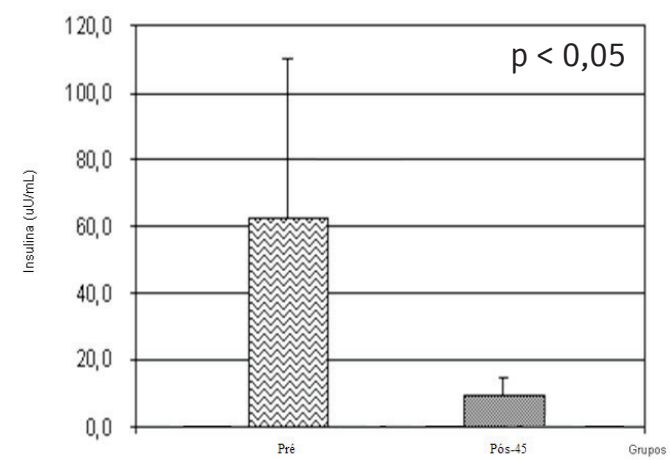

\section{CONCLUSÃO}

Ao final, o acompanhamento dos níveis plasmáticos de IGF-1, glicose e insulina no pré e pós-operatório precoce de pacientes submetidos à cirurgia de gastroplastia e derivação em $Y$ de Roux por laparoscopia, na forma como foi aqui conduzida, permitiu con-

cluir que os níveis plasmáticos de IGF-1, insulina e glicose reduziram após a referida intervenção cirúrgica. Sugere-se ainda o acompanhamento de tais pacientes para observação da manutenção ou alteração nesses parâmetros. 


\section{REFERÊNCIAS}

BARRETO-FILHO, JAS, OLIVEIRA JLM, SANTOS CM, AGUIAR-OLIVEIRA MH. Papel do eixo GH/IGF1 na fisiopatologia da síndrome metabólica: resistência insulínica e lesão de órgão alvo. Rev Bras Hipertens, 2005, 12:159-164.

BROLIN RE. Bariatric surgery and long-term control of morbid obesity. JAMA 2002, 288:2793-2796.

BUCHWALD H, AVIDOR Y, BRAUNWALD E, JENSEN MD PORIES W, FAHRBAC $K$, et al. Bariatric surgery: a systematic review and meta-analysis. JAMA 2004, 292:1724-1737.

CARVALHO PS, MOREIRA CLCB, BARELLI, MC, OLIVEIRA FH, GUZZO, MF, MIGUEL GPS, et al. Cirurgia bariátrica cura síndrome metabólica? Arq Bras Endocrinol Metab, 2007, 51:79-85.

COHEN RV, PINHEIRO-FILHO C, SCHIAVON CA, CORREA JLL. Alterações sistêmicas e metabólicas da cirurgia laparoscópica. Rev Bras Videocir, 2003, 1:77-81.

CONCEIÇÃO FL, BOGUSZEWSKI CL, MEISTER LHF, ZANINELLI DCT, RADOMINSKI, RB, KNOEPFELMACHER, M, et al. Deficiência de GH en adultos: resultado do estudo multicêntrico brasileiro. Arq Bras Endocrinol Metab, 2003, 47:321-22.

ENGSTROM B, BURMAN P, HOLDSTOCK C, ÖHRVALL $M$, SUNDBOM M, KARLSSON FA. Effects of gastric bypass on the GH/IGF-1 axis in severe obesity and a comparison with GH deficiency. 2006, 154:53-9.

FEITOSA ACR, MANCINI MC, CERATO C, VILLARES S, HALPERN A. Relação entre o perfil metabólico e níveis de leptina em indivíduos obesos. Arq Bras Endocrinol Metab, 2007, 51:59-64.
FREIRE RF. Avaliação dos níveis de IGF-1, glicemia e insulina em pacientes obesos mórbidos submetidos à cirurgia bariátrica [Dissertação]. Aracaju (SE): Universidade Federal de Sergipe; 2006.

FRÜBECK G, ROTELLAR F, HERNÁNDEZ-LIZOAIN JL, GIL MJ, GÓMEZ-AMBROSI J, SALVADOR J, et al. Fasting ghrelin concentrations 6 months after gastric bypass are not determined by weight loss or changes in insulin. Obes Surg., 2004, 14:1208-15.

GELONEZE B, PAREJA JC. Cirurgia bariátrica cura a síndrome metabólica? Arq Bras Endocrinol Metab, 2006, 50:400-7.

GELONEZE B, TAMBASCIA MA, PAREJA JC, REPETTO EM, MANGA LA, PEREIRA SG. Serum leptin levels after bariatric surgery across a range of glucose tolerance from normal to diabetes. Obes Surg, 2001, 11:693-8.

GOHIER B, RICHARD-DEVANTOY S, DENĖS D. Le psychiatre et la chirurgie bariatrique. Rev Psych, 2010, 168:220-223.

HALPERN ZSC, RODRIGUES DB, COSTA RF. Determinantes fisiológicos do controle do peso e apetite. Rev Psiq Clin, 2004, 31:150-153.

HALPERN A, MANCINI MC, CERCATO C, VILLARES SMF, COSTA APAC. Efeito do hormônio do crescimento sobre parâmetros antropométricos e metabólicos na obesidade andróide. Arq Bras Endocrinol Metab, 2006, 50:68-73.

JAUNOO SS, SOUTHALL PJ. Bariatric surgery. Inter J Surg 2010;8:86-9.

GELONEZE B, TAMBASCIA MA, PAREJA JC, REPETTO EM. The insulin tolerance test in morbidly obese patients undergoing bariatric surgery. Obes Res, 2001, 9:763-769. 
LEAL-GUADARRAMA LI, OCHOA-ROJAS M, MÉNDEZ JD Importancia clínica de los factores de crescimiento parecidos a la insulina. Gac Med Mex, 2003, 139:589-598.

MAGGARD MA, SHUGARMAN LR, SUTTORP M, MAGLIONE M, SUGERMAN HJ, LIVINGSTON EH, et al. Meta-analysis: surgical treatment of obesity. Annals of Internal Med, 2005, 142:547-559.

MANCINI MC, HALPERN A. Pharmacological treatment of obesity. Arq Bras Endocrinol Metab, 2006, 50:377-589.

MÁRQUEZ-LOPES Z. Qualidade de vida em pacientes portadores de obesidade mórbida submetidos à gastroplastia vertical com banda com derivação em $Y$ de Roux [Dissertação]. Santa Catarina: Universidade do Oeste de Santa Catarina; 2005.

MARTINS MVD. Porque o “bypass” gástrico em Y de Roux é atualmente a melhor cirurgia para o tratamento da obesidade. Rev Bras Videocir, 2005, 3:102-104.
MOTA GR, ZANESCO A. Leptina, grelina e exercício físico. Arq Bras Endocrinol Metab, 2007, 51:25-33.

MAURAS N, MARTINEZ V, RINI A, GUEVARA-AGUIRRE $J$. Insulin-like growth factor I and growth hormone (GH) treatment in GH deficient humans: differential effects on protein, glucose, lipid and calcium metabolism. JCEM 2000, 85:1686-1694.

NOWICKI T, BURNS C, FULBROOK P, JONES J. Changing the mindset: an interdisciplinary approach to management of the bariatric patient. J Royal Col Nurs Aus, 2009, 16:171-175.

PORTO MCV, BRITO IC, CALFA ADF, AMORAS M, VILLELA, N, ARAÚJO LBM. Perfil do obeso classe III do ambulatório de obesidade de um hospital universitário de Salvador - Bahia. Arq Bras Endocrinol Metab, 2002, 46:668-673.

TORPY J, BURKE A, GLASS M. Surgery bariatric. JAMA 2005, 1:294.

TERNOVITS CA, TICHANSKY DS, MADAN AK. Band versus bypass randomization and patients choices and perceptions. Surg Obes Rel Disease, 2005, 2:6-10. 